4. Glycogen $\mathrm{D}$ bildet sich, wenn Thiere allein mit gereinigtem Käse gefüttert werden. Seine spec. Drehung ist $(\alpha) \mathrm{j}=+211^{\circ}$. $100 \mathrm{ccm}$ Wasser von $100^{\circ}$ lösen davon 2,7 grm.

Ein so greller Unterschied der Eigenschaften genügt vollkommen zur Annahme mehrerer Modificationen des Glycogens. $\mathrm{Ob}$ indess vier oder noch mehr Modificationen bestehen, lässt sich nicht mit Bestimmtheit sagen."

\title{
Beiträge zur Lehre vom künstlichen Diabetes ${ }^{1}$ ).
}

\author{
Von
}

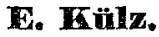

Die Angabe Bernard's'), dass Reizung der centralen Vagusenden Diabetes hervorrufen könne, ist trotz ihrer Tragweite für die Pathogenese des Diabetes lange Zeit unberïcksichtigt geblieben. Erst vor Kurzem hat Eck hard ${ }^{g}$ ) gelegentlich seiner Untersuchungen über die Stellung des Nervensystems zum Diabetes die Aufmerksamkeit auf dieselbe wieder gelenkt und sie zugleich einer eingehenden Prüfung am Kaninchen unterzogen, welche um so nothwendiger war, als Bernard's Angabe sich nur auf Versuche an Hunden stütat ${ }^{4}$ ). Die ersten Versuche richtete Eckhard so ein, dass er das centrale Ende nur eines in der Höhe der Mitte des Halses durchnittenen Vagus reizte, während der andere intact war, und zwar so, dass die Reizung unmittelbar nach der Darch-

1) Zum Theil nach Versuchen, die in Gemeinschaft mit Dr. J, P. F. Richter (vergl. dessen Inang:-Dissert. Marburg 1878) angestellt wurden.

2) Cl. Bernard, leçons sur la physiologie et la pathologie du système nervenx. T. IL. 1858. p. 442.

3) C. Eckhard, Beiträge zur Anatomie u. Physiologie 8, 94.

4) Bekanntlich liegen Beobachtungen vor, die beweisen, dass sich Hund und Kaninchen in dieser Beziehung verschieden verhalten können. So kann man z. B. durch Verletzung des Unterwurms beim Kaninchen Diabetes erzeugen, nicht aber beim Hund. 
schneidung begann und während einer Stunde so fortgesetzt wurde, dass die ein bis zwei Minuten langen Reizungen durch mehrere Minuten andauernde Pausen unterbrochen wurden. In allen diesen Fällen fand sich nach einstïndiger Reizung oder doch in der zweiten Stunde nach der Reizung ein Diabetes vor, der gewöhnlich ein bis zwei Stunden, manchmal anch etwas länger anhielt. Die Frage, ob schon die einfache Vagusdurchschneidung Diabetes erzeugen könne, ist von einigen Autoren bejaht, von andern verneint worden. Eckhard sah sich veranlasst, auch diese Angaben besonders zu prüfen. Von seinen Versuchen theilt er zwei, mit allen Cautelen angestellte, ausfïhrlich mit. Bei einem Thiere wurde der linke, bei dem andern beide Vagi durchschnitten. Der Harn wurde in beiden Fällen sehon eine Stunde nach der Durchschneidung zuckerhaltig. Eckbard weist uibrigens darauf hin, dass bei schlecht genährten, herabgekommenen Thieren der Diabetes ausbleiben könne, ja dass man den Zucker übersehen könne, weil er oft nur in kleinen Mengen und nur in der ersten Stunde nach der Durchschneidung rorhanden ist. Die erstgenannten Reizungsversuche mussten bei dieser Sachlage nunmehr wiederbolt werden. Deun es ist klar, dass alle diejenigen Versuche nicht beweiskräftig sind, in denen man vor der Reizung des centralen Vagusendes nicht den Effect der einfachen Durchsehneidung abgewartet hat. Eckhard liess daher in seinen weiteren Versuchen nach der Section eines Vagus eine Stunde verstreichen und begann dann in Fällen, wo kein Diabetes auftrat, sofort mit der Reizung der centralen Vagusenden; in Fällen jedoch, in denen sich Diabetes einstellte, wartete er, bis der Zuckergehalt des Harns kaum noch bemerkbar war und schritt erst dann zur Reizung. In Fällen der ersteren Art erhielt er fast ausnahmslos Diabetes, in Fällen der letzteren Art zwar nicht immer, aber doch sehr oft, namentlich, wenn die Thiere kräftig waren und das Verschwinden des durch die einfache Vagusdurchschneidung erzeugten Diabetes sich nicht zu lange hinzog. Einen besonders instructiven Versuch theilt er ausführlicher mit. Einem Kaninchen wurde der linke Vagus durchschnitten, nach einer Stunde war der Harn zuckerhaltig. Er reizte nun das centrale Ende des linken Vagus während $3 / 4$ Stunden, in Zeiträumen von 5 zu 5 Minuten jedesmal $1 \frac{1}{2}$ Minute. Der eine Stunde nach Beginn der Reizung ausgedrückte Harn war stark zuckerhaltig; 
auch die in den drei nachfolgenden Stunden gesammelten Harnmengen mussten noch als zuckerhaltig bezeichnet werden. Am folgenden Morgen war der Harn zuckerfrei. Nach 3/4 stiindiger Reizung trat von Neuem ein mehrere Stunden anhaltender Diabetes auf. So behandelte Eckhard das Thier noch mehrere auf einander folgende Tage. Morgens enthielt der Harn keinen Zucker, nach der Reizung trat er wieder mehrere Stunden hindurch auf. Eckhard fasst sowohl den durch einfache Vagussection als den durch Reizung des centralen Vagusendes hervorgerufenen Diabetes als reflectorisch ${ }^{1}$ ) auf.

Im Hinblick auf den Uebelstand, dass das Gebiet des Diabetes überreich an theils unbewiesenen, theils vagen Theorien, arm dagegen an hinreichend sichergestellten Thatsachen ist, auf Grund deren sich weiter operiren liesse, bedarf es wohl kaum einer Entschuldigung, dass wir die vorausgeschickten, die Stellung des Nervus vagus zum Diabetes betreffenden Angaben einer nochmaligen methodischen Prüfung unterzogen. $\mathrm{Zu}$ allen in der nachfolgenden Arbeit überhaupt zur Sprache kommenden Versuchen wurden nur kräftige, ausgewachsene Kaninchen verwandt. Die zum Nachweis des Zuckers im Harn benutzte Fehling'sche Lösung wurde in Form zweier Lösungen (Kupferlösung - Seignettesalzlösung) angefertigt und aufbewahrt. Unmittelbar vor dem Gebrauch wurde die Mischung hergestellt und ein Theil derselben nach bekannter Weise auf ihre Haltbarkeit geprüft. Weibliche Thiere wurden möglichst, trächtige gänzlich ausgeschlossen, $d a$ bei dem Ausdrücken des Harns bei letzteren leicht Blutungen erfolgen und der Harn tragender Kaninehen ganz ähnlich reducirende Eigenschaften besitzt, wie der Harn schwangerer Frauen. In allen Fällen wurde übrigens eine controlirende Prüfung mit der Trommer'schen Probe vorgenommen. Der Harn wurde unmittelbar vor dem Eingriff, nach dem Eingriff stündlich ausgedrückt. Die einzelnen Harnproben wurden möglichst gleichmässig auf Zucker geprüft und stets etiquettirt aufbewahrt, so dass man am Schluss des Versuches noch einmal vergleichsweise den Ausfall aller Reactionen iuberblicken konnte. Die Reizung wurde mit dem du Bois'schen Schlittenapparat in der von Eekhard angegebenen Weise aus-

1) S. Landois, Lehrbuch der Physiologie. S. 269. 
geführt. Von vornherein sei hervorgehoben, dass wir die Richtigkeit aller von Eekhard gemachten Angaben bestätigen können. Wir betrachten es als eine Zufälligkeit, dass die von Eckhard mitgetheilten Belege immer nur den linken Vagus betreffen. Da bekanntlich manche Autoren beide Vagi in ihrer Wirkung z. B. auf das Herz nicht gleichwerthig erachten, so haben wir den zuletzt mitgetheilten iustructiven Versuch Eckhard's am rechten Vagus wiederholt und zwar mit ganz denselben Resultaten. Uebrigens scheint es einer so lange fortgesetzten intermittirenden Reizung nicht zu bedürfen, denn es gelang uns schon durch eine 5 Minuten lang andauernde Reizung ohne Steigerung der Stromstärke unverkennbaren Diabetes hervorzurufen.

Des Hinweises scheint es uns immerhin werth zu sein, dass in der Diabetesliteratur einige Fälle [A nger ${ }^{1}$ ), Percy ${ }^{2}$ ), Henrat ${ }^{3}$ )] existiren, in denen man bei der Section den Nervus vagus entartet fand. In Ang er's Fall "wies die Section ein mandelgrosses Kalkconcrement am N. vagus neben zerstreuten Lungentuberkeln nach".

Percy fand "das Ganglion semilunare und die Nervi splanchnici sowie den Nervus vagus verdickt und von knorpelartiger Härte". In Henrat's Fall war das Hauptergebniss der Section "die Auffindung eines haselnussgrossen Tumors am rechten Nervus vagus, da wo er den Hilus der Lunge kreuzt. Seine Oberfläche war höckrig, umgeben war er von einer harten Schale, welche sandige Granulationen und etwas käsiges Material einschloss. Der Nervenstamm verlor sich vollständig in dieser Oberfläche, er verliess die Geschwulst mit geschmälertem Volumen; erst einige Centimeter abwärts wurde dasselbe wieder normal".

Uebrigens habe ich die Versuche noch in der Weise modificirt, dass ich den $\mathrm{N}$. vagus nach seinem Durchtritt durch das Zwerchfell mit möglichster Schonung der Leber aufsuchte und den Effect der Durchschneidung wie der Reizung des centralen Endes beiderseits mehrfach studirte. Die Resultate waren den am Hals-

1) Beiträge zur Balneologie. Aus den Kurorten Böhmens herausgegeben von mehreren Vertretern derselben unter der Redaction des Landesmedicinalrathes Dr.Löschner. Bd. 1. 1863. Dr. Anger, Carlsbad in seinen Beziehungen zum uropoetischen System.

2) v. Ziemssen's Handbuch der spec. Pathol. u. Therapie. Bd. XIII. 2. Hälfte 1876. Diabetes mellitus u. insipidus von H. Senator. S. 140.

3) Centralblatt für d. med. Wissensch. 1876. Nr. 12. S. 223. H. Hen rat, Diabète; tumeur sur le trajet du pneumogastrique. Gaz. hebdom. 1875. Nr. 35 . 
vagus gewonnenen vollkommen analog. Durch diese Versuche dürfte die schon mehrfach ausgesprochene reine Vermuthung, dass der Diabetes auf Innervationsstörungen des Vagus zurückzufïhren, und dass mehrere Symptome desselben durch Ueberreizung der Terminalzweige des Vagus im Magen bedingt seien, doch wenigstens eine thatsächliche Stütze gewinnen, so dass die Möglichkeit einer solchen Entstehungsweise des Diabetes nicht unbedingt in Abrede gestellt werden kann.

Filehne ${ }^{1}$ ) reizte bei Kaninchen „das centrale Ende des (linken) Depressor alle 5 Minuten während $1 / 2$ Minute mit mässigen Inductionsströmen. Nach 20-45 Minuten enthielt der Urin Zucker". Eckhard ${ }^{2}$ ) und ich können auf Grund eigener Versuche die Richtigkeit dieser Angaben bestätigen. Eine andere Behauptung Filehne's, dass die Meliturie ausbleibt, „wenn das (linke) centrale Vagusende aber excl. Depressor eine Stunde hindurch gereizt wird", stimmt nicht mit den von Eckhard und uns erhaltenen Resultaten überein. Es dürfte gar nicht leicht zu beweisen sein, dass in Filehne's Versuch die centrale Erregung des Depressor die Ursache des Diabetes ist. Es könnte die Reizung dieses Nerven sich paradox auf den Vagus übertragen. Dass eine centrale Reizung des Vagus und nicht etwa eine paradoxe Uebertragung auf den Depressor in Eckhard's und unsern Versuchen die Ursache ist, geht auch daraus hervor, dass die blosse Durchschneidung wirksam sein kann. Dafür sprechen auch die Versuche, in denen wir den Vagus am Magen durchschnitten und reizten, vorausgesetzt, dass die längere Blosslegung der Eingeweide hier ganz unschädlich ist ${ }^{3}$ ).

1) Filehne, Melliturie nach Depressor-Reizung beim Kaninchen. Centralblatt f. d. med. Wissensch. 1878 Nr. 18.

2) Nach mündlicher Mittheilung.

3) Laffont (Recherches sur la vascularisation du foie et des viscères abdominaux; au point de vue de la production du diabète par influence nerveuse. Progrès méd. 1880. Nr. 10) fand, dass eine lange fortgesetzte Reizung der centralen Stïmpfe der Nerv. depressores bei Kaninchen Meliturie zur Folge hat und dass auch nach centraler Vagusreizung bei nicht curaresirten Thieren eine, aber geringere Meliturie eintritt, die er als Folge der Respirationsstörung betrachtet. (Centralbl. £. d. med. Wịsensch. 1880. S. 515.) 
Pavy ${ }^{1}$ sah zuckerhaltigen Harn nach Injection von Phosphorsäure in das allgemeine Venensystem und ebenso nach Einfiibrung derselben in den Darmkanal auftreten. Die Säure wirkt nach Pavy dadurch, „dass sie die Constitution des Blutes verändert und dasselbe ungeeignet macht, zu der normalen Ausführung der in der Leber vorgehenden Operation beizutragen. " Mit dieser Erklärung, wenn man sie als solche überhaupt ansehen will, dürtte wenig gewonnen sein.

Goltz ${ }^{2}$ ) injicirte Kaninchen Milchsäure ( 50 pCt Lösung) per os in den Magen. "Es trat darnach in vielen Fällen nach längerer oder kürzerer Zeit Zucker im Harn auf, der in einem Fall $4,9 \mathrm{pCt}$ betrug. Die Harnmenge fand sich dabei nicht vermehrt. Die Thiere nahmen in der Zwischenzeit in Folge der heftigen Gastritis keine Nahrung zu sich." Ueber die Bedingungen, unter denen diese Meliturie auftrat, schienen ihm die Experimente folgendes zu ergeben: „Die Milchsäure muss längere Zeit auf den Organismus wirken können; daher zeigen sich zu grosse oder zu starke Dosen von Milchsäure unwirksam, ihnen erliegt das Thier zu schnell. Wurden pro dosi $10-12 \mathrm{ccm} 50 \mathrm{pCt}$ Milchsäure-Lösung täglich einmal gegeben, so zeigte sich der erste Zucker 36-48 Stunden nach der ersten Injection. Kleinere Dosen zeigten sich ebenfalls unwirksam. Ein Theil der eingeführten Milchsäure liess sich im Harn nachweisen und zwar, wie sie eingeführt war, als gewöhnliche, nicht als Fleischmilchsäure." Die am Schlusse der Mittheilung in Aussicht gestelite Fortsetzung der Untersuchung „ob auch andere Säuren dieselbe Wirkung haben, ob die verminderte Alkalescenz des Blutes, oder eine Ueberladung desselben mit einem leicht oxydirbaren organisehen Körper die Ursache der Zuckerausscheidung durch die Nieren sei", scheint, da keine weitere Publikation vorliegt, nicht ausgefiuhrt worden zu sein.

Gelegentlich seiner Untersuchung über Icterus fand Naunyn ${ }^{3}$ )

1) Pavy, Untersuchungen über Diabetes mellitus, dessen Wesen and Behandlung. Deutsch von Langenbeck 1864. S. 77.

2) Centralblatt f. d. med. Wissensch. 1867. Nr. 45. Meliturie nach Milchsäureinjection. Vorläufige Mittheilung von G. Goltz.

3) Naunyn, Beiträge zur Lehre vom Icterus. Reichert's u. du BoisReymond's Archiv 1868, S. 413 u. 414. 
in dem Harn eines Hundes, dem er 5,0 ccm conc. roher Salzsäure, auf $100,0 \mathrm{ccm}$ verdünnt, mittelst der Schlundsonde in den Magen gespritzt hatte, ziemlich viel Eiweiss und 1 pCt Zucker (nach dem Enteiweissen mit Fehling'scher Lösung titrirt). Ausserdem enthielt der Harn mit Fettlkörnchen besetzte Cylinder.

Diese Beobachtungen von Pavy, Goltz und $\mathrm{Nauny} n$ haben das Gemeinsame, dass der Diabetes nach Zufuhr von Säuren auftrat. Da die Angaben von Keinem bisher geprifft wurden, so entschloss ich mich, diese Versuche am Kaninchen zu wiederholen, um so mehr, als die Möglichkeit nicht ausgeschlossen war, dass der durch die Säuren hervorgerufene Diabetes auf reflectorischem Wege, d. h. dureh Reizung der Vagusendigungen im Magen zu Stande gekommen sei.

Von der Phosphorsäure (spec. Gew. 1,12) wurden in allen Versuchen 5,0 grm mit $25,0 \mathrm{ccm}$ Wasser verdünnt, durch die Schlundsonde in den Magen injicirt. Diese Dosis hatte sich nach einigen Vorversuchen als diejenige erwiesen, welche von den Thieren relativ gut vertragen wird und am sichersten zum Ziele führt. 'Zwei Stunden nach der Injection wird meist der Harn zucker- aber anch eiweisshaltig. Der Eiweissgehalt ist von Pavy übersehen resp. nicht angegeben worden. Bei mikroskopischer Untersuchung fanden sich stets im Harn Cylinder and verfettete Epithelien. Die Ausscheidung von Zucker und Eiweiss kann 30 Stunden deutlich andauern. Am dritten Tage konnte der Harn der Thiere, die nun auch wieder frassen, als normal bezeichnet werden. Kräftige Thiere können also den Eingriff überleben.

Von der Milchsäure (spec. Gew. 1,12) wurden auf Grund einer Reihe von Versuchen stets 5,0 grm mit 25,0 ccm Wasser verdünnt in den Magen injicirt. Trotz gleicher Experimentationsweise ist die Reaction der Thiere recht verschieden. Die einen starben sehon zwei Stunden nach der Injection, andere nach 5 und mehr Stunden, noch andere überlebten den Eingriff und erholten sich wieder vollständig. Der Harn aller Versuchsthiere enthielt ausnahmslos schon in der zweiten Stunde nach der Injection Eiweiss, ein Punkt, der von Goltz nicht bericksichtigt worden zu sein scheint. Der Harn enthielt ferner ausnahmslos Cylinder und verfettete Epithelien. Die Zuekerausscheidung war in in den verschiedenen Versuchen keineswegs von gleicher Intensität; sie kann 
schon eine Stunde nach dem Eingriff durchaus deutlich auftreten und zwei Tage hindurch anhalten. In dieser Beziehung unterscheiden sich also unsere Versuchsresultate wesentlich ron denen von Goltz, der die Znckerausscheidung erst 36-48 Stunden nach der Injection beginnen lässt. Wenn auch Eiweiss- and Zuckerausscheidung nicht streng parallel gehen, so ist das Auftreten wie das Aufhören derselben im Allgemeinen doch meist gleichzeitig.

Die Salzsäure kam in Form der verdünnten $\mathrm{HCl}$ zur Verwendung und zwar wurden $5,0 \mathrm{cem}$ mit $20 \mathrm{ccm}$ Wasser weiter verdünnt und in den Magen injicirt. Schon nach einer Stunde trat Eiweiss- und Zuckerausscheidung auf und zwar uiberdauerte die erstere die letztere. Der Harn enthielt wieder Cylinder und verfettete Epithelien. Noch am zweiten Versuchstag liess sich im Harn Zucker nachweissen, wenn auch in geringerer Menge. Es stimmen somit unsere Resultate vollkommen mit der Beobachtung von Naunyn überein. Die Thiere überlebten alle den Eingriff, mehrere wurden 5 Tage hindurch beobachtet. Der Harn der überlebenden Thiere, die übrigens schliesslich wieder frassen, konnte am vierten Versuchstage als normal bezeichnet werden.

Der durch jede der drei Säuren hervorgerufene Diabetes stimmt insofern mit dem Vagus-Diabetes überein, als die Zuckerausseheidung schon verhältnissmässig früh auftritt. Die heftige Gastritis, welche bei den Thieren, wie einige Sectionen ergaben, auftritt, könnte ferner recht wohl als Reiz für die Vagusendigungen im Magen angesprochen werden. Allein der Umstand, dass bei dem Säure-Diabetes im Harn zugleich Eiweiss in erheblicher Menge auftritt, sowie die Verfettungen, welche sich in verschiedenen Organen (Epithelien der Labdritsen, Leberzellen, Epithelien der g'ewundenen Harnkanälchen) nachweisen lassen ${ }^{1}$ ), dürften doch gegen die Auffassung des Säure-Diabetes als durch Vagusreizung bedingt sprechen.

Es kam uns nun in weiteren Versuchen darauf an, eine Substanz aufzufinden, die, in den Magen eingefuibrt, denselben kräftig reizte, ohne jedoch Verfettung in den Organen, sowie Eiweissausscheidung durch den Harn zu veranlassen. Am geeignetsten erschien uns das Kochsalz, zumal, da durch die Versuche von Bock

1) Vergl. auch in dieser Beziehung das Original der Naunyn'schen Arbeit. 
und $\mathrm{Hoffmann}{ }^{1}$ ) einerseits und mir $^{2}$ ) andererseits bekannt ist, dass, wenn man eine 1proc. Kochsalzlösung continuirlich in das Gefässsystem einleitet, Diabetes auftritt. Allen Thieren wurde dieselbe Menge Kochsalz (5,0 grm) in den Magen eingeführt. In einigen Versuchen wurde dasselbe in $20,0 \mathrm{cem}$, in anderen in $30,0 \mathrm{eem}$ Wasser gelöst. Schon im Verlauf der ersten Stunde nach der Injection trat eine Polyurie auf, die sich noch steigerte und mehrere Stunden anhielt. Der Harn enthielt, wenn überhaupt, nur Spuren von Eiweiss, dagegen keinen Zucker. Einige von den Thieren starben am zweiten Tage, andere am vierten. Die Magenschleimhaut war stark ödematös, stellenweise schwarzbraun, brandig. Es dürften somit auch diese Versuche, bei denen es an Reizung der Magenschleimhaut resp. der Vagusendigungen nicht fehlte, in gewisser Beziehung gegen die Auffassung des Säure-Diabetes als reflectorisehen mitsprechen.

Sehiff') hat die Beobachtung gemacht, dass nach Durchschneidung des Nervus ischiadicus Diabetes entsteht. Die Angabe ist bereits ohne nähere Prüfung in mehrere Schriften ${ }^{4}$ ) übergegangen. Schiff stellt sich die Genese dieses Diabetes folgendermassen vor: Der Ischiadicus führt eine grosse Zahl von Gefässnerven; diese werden nach der Durchschneidung gelähmt. In den gelähmten Gefässen der Extremität entsteht sodann ein Ferment, welches durch die Cireulation in die Leber gelangt und hier eine abnorme Zuckerbildung einleitet.

Durch 10 an ausgewachsenen und wohlgenährten Kaninchen angestellte Versuche, in denen bald der rechte, bald der linke Ischiadicus durchschnitten wurde, konnten wir mit einer Ausnahme die Richtigkeit der Schiff'schen Angaben bestätigen. Der Zucker kann schon eine Stunde nach der Durchschneidung auftreten. In den Fällen, wo er nach der ersten Stunde nur andeutungsweise

1) Bock u. Hoffmann, Reichert's u. du Bois-Reymond's Archiv 1871. Heft 6.

2) Külz, Beiträge zur Hydrurie u. Melitarie. Habilitationsschrift 1872.

3) M. Schiff, Nouvelles recherches sur la glycogénie animale, lecons faites au muséum de Florence en Janvier 1866. p. 379 und Journàl de l'anatomie et de la physiologie 1866. T. III. p. 354.

4) Eulenburg u. Guttmann, die Pathologie des Sympathicus auf physiologischer Grundlage. 1873. S. 188. 
auftrat, haben wir ihn in der zweiten Stunde nie vermisst. Die Reaction tritt in verschiedenen Versuchen bald stäker, bald schwächer auf, kann aber bei einiger Aufmerksamkeit nicht wohl übersehen werden, wenn die Versuchsthiere genügend kräftig sind. Die Dauer der Zuckerausscheidung ist ebenfalls versehieden; im Durchschnitt erstreckt sie sich nur auf 2-3 Stunden, so dass in den letzien Harnproben nur sehr geringe Mengen vorhanden sind. In einem Fall hielt übrigens die Zuckeransscheidung noch am dritten Tage an und zwar wurde in diesem Fall die Prïfung der einzelnen Harnproben ganz besonders sorgfältig vorgenommen.

Der von Scbiff iiber die Entstehnng des Diabetes gegebenen Erklärung können wir nicht beipflichten. Zu ihrer Prufung leiteten wir in den einen Versuchen filtrirten menschlichen gemischten Speichel, in den andern dureh Extraction von Rindspancreas mittelst Glycerin gewonnene Pancreasdiastase in die Vena jugularis externa. Bei beiden Versuchsformen konnte in dem Harn kein Zucker nachgewiesen werden, obwohl, wie wir uns iberzeugt hatten, Speichel wie Pancreasdiastase aus Kaninchenleber dargestelltes Glycogen energisch saccharificirten. Hervorgehoben sei noch, dass auch zu diesen Versuchen nur kräftige und wohlgenährte Thiere benutzt wurden. Das saccharificirende Ferment des Speichels wie des Pancreas ging ubrigens in den Harn iber. Schon in der zweiten Stunde nach dem Beginn der Einleitung konnten sie im Harn durch Stärkekleister nachgewiesen werden, so jedoch, dass die letzteren Harnproben im Vergleich zu den ersteren eine geringere Fermentwirkung zeigten. Uebrigens sei noch bemerkt, dass sowohl der Speichel wie das Pancreasdiastasehaltige Glycerin vor jedem Versuch mit negativem Resultat anf Zucker geprüft wurden.

Die Schiff'schen Angaben gewinnen dadurch ein erneutes Interesse, dass man bei Ischias mehrmals nicht unerhebliche Mengen von Zucker im Harn gefunden hat. So betrug der Zuckergehalt in 4 von $\mathrm{Braun}^{1)}$ beobachteten Fällen $1 / 2-2 \frac{1}{2}$ pCt. Eulenburg und Guttmann ${ }^{2}$ ) haben in 2 Fällen diese Beobachtung bestätigt. „In dem ersten Falle war allerdings der Zuckergehalt ein so geringer, dass das pathologische dieses Falles nicht mit Sicher-

1) Braun, Balneotherapie. 3. Aufl. pag. 411. 1868.

2) Eulenburg u. Guttmann, Die Pathologie des Sympathieus auf physiologischer Grundlage. 1873. S. 194. 
heit behauptet werden konnte. In einem zweiten Falle aber von inveterirter rechtsseitiger Ischias liess sich vorübergehend ein Zuckergehalt von ca. 1 pCt bei 1,023 spec. Gew. und einer täglichen Harnmenge von $3000 \mathrm{ccm}$ nachweisen."

Ich habe in 13 Fällen von Ischias auf diesen Punkt geachtet. Mehrmals konnte ich stärkere, schon bei mässigem Erwärmen auftretende Reduetionen, wie man sie bei normalem Harn zu beobachten nicht gewohnt ist, constatiren. Es gelang mir jedoch in keinem Falle, eine Drehung des mit Bleizucker entfärbten Harns mit voller Sicherheit nachzuweissen. $O b$ die Bestimmungen von Braun einerseits, von Eulenburg und Guttmann andererseits durch Polarisation controlirt oder vielleicht nur durch Polarisation gewonnen sind, lässt sich aus dem Original nicht ersehen. Die alleinige Bestimmung durch Drehung würde jedenfalls wichtiger sein, als die alleinige Bestimmung durch Kupferlösung.

E r $b^{1}$ ) äussert sich folgendermassen: „Bei Diabetes sol Ischias, wie ich von befreundeter erfahrener Seite (Friedreich?) weiss, nicht selten vorkommen, während amgekehrt Braun in seiner Balneotherapie (3. Aufl. pag. 411) angibt, dass er öfter Diabetes als Symptom von Ischias beobachtet habe." Da wir die Schiff'sche Erklärung nach unserer experimentellen Prüfung nicht für begründet erachten konnten, so drängte sich uns im Anschluss an die Versuche von Bernhard, Eckhard und uns ïber reflectorischen Diabetes die Vermutbung auf, dass möglicherweise die Zuckerausscheidung nach Ischiadicus-Durchschneidung in derselben Weise zu. deuten sei, wie der Diabetes nach Vagussection. Dieser Gedanke hat nichts Absurdes, insofern nach ïbereinstimmenden Angaben von 0 wsjannikow und Tschiriew ${ }^{2}$ ), Griitzner ${ }^{3}$ ) und mir ${ }^{4}$ ) Reizung des centralen Endes vom Nervus ischiadicus die Speichelsecretion beschleunigt unter Vermittlung des am Boden des

1) Erb, Handbuch der Krankheiten des Nervensystems. II. Erste Hälfte. S. 154 (S. Ziemssen, Handbuch der spec. Pathologie u. Therapie. XII. Firste Hälfte. 1876).

2) Mélanges biologiques tirēs du Bulletin de l'académie impériale des sciences de St. Petersbourg. T. VIII.

3) Pflüger's Archiv Bd. VII. S. 522 u. ff. 1873.

4) E. Külz, Beiträge zur Pathologie und Therapie dẹs Diabetes mellitus und insipidus. Bd. II. S. 16 ๗. 17. 1875. 
vierten Ventrikels unweit des Diabetescentrums gelegenen Speichelcentrums ${ }^{1)}$ [Bernard, Eckhard ${ }^{2}$ ) Lö $b^{3}$ ), Grützner ${ }^{4}$ ) und ich ${ }^{5}$ )].

Um die Richtigkeit oder Unrichtigkeit dieser Deutung zu erweisen, wurden Versuche ganz in derselben Weise eingerichtet, wie wir sie oben beim Vagus geschildert haben. Es wurde zunächst der Ischiadicus durchschnitten und der Effect der Durchschneidung bis zum Verschwinden der Zuckerreaction abgewartet; sodann wurde das centrale Ende des Ischiadicus in derselben Weise wie dss centrale Vagusende electrisch gereizt. Jede einzelne Reizung dauerte $1 \frac{1}{2}$ Minute, so dass nach Intervallen von 6-8 Minuten der Nerv innerhalb einer Stunde 6-7mal gereizt wurde. Die Stromstärke war in allen Versuchen die gleiche und dieselbe, deren wir uns bei Reizung des Vagus bedient hatten. Der zwei Stunden nach Schluss der Reizung ausgedrückte Harn zeigte in allen Versuchen deutliche Reduction und zwar meist stärker als nach einfacher Durchschneidung. In den folgenden Harnproben nahm die Reduction wieder ab. Somit erseheint uns die oben angegebene Deutung wenigstens zulässig ${ }^{6}$ ).

1) Hervorgehoben sei hier, dass Friedreich bereits 1863 in seiner Arbeit über degenerative Atrophie der spinalen Hinterstränge (Virchow's Archiv Bd. 26) einen Fall von Ataxie mittheilte, in welchem successive Diabetes, Ptyalismus, Schweisssucht und excessive wässerige Stuhlentleerungen auftraten, und schon damals zu der Anschauung gelangte, dass im verlängerten Mark resp. Boden des 4. Ventrikels secretorische Centren gelegen seien. In seinem Buch über progressive Muskelatrophie (Berlin 1873) hat F. diesen Punkt S. 183 noch ausführlicher erörtert.

2) Eckhard, Beiträge zur Anatomie a. Physiologie. Bd. IV. S. 191. 1869.

3) Lo eb, Eckhard's Beiträge. Bd. V. S. 3.

4) 1. c.

5) $1 . \mathrm{c}$.

6) Nach Adolph Jänicke (Untersuchungen über die Secretion der glandula parotis. Pflüger's Archiv 17. 183) braucht übrigens die Vermehrung der Speichelsecretion nach Reizung des Ischiadicus nicht als Reflexvorgang aufgefasst zu werden. Die hierauf bezüglichen Stellen seiner Arbeit sind: „dass die Reizung sensibler Nerven z. B. des Ischiadicus, der Magenschleimhaut Speichelsecretion hervorruft, erklärt sich dadurch, dass durch sie eine allgemeine Gefässverengerung bewirkt wird" ... . „Es ist wohl nicht zu gewagt anzunehmen, dass manche Erscheinungen, die wir jetzt zu den Hemmungs- und Reflexvorgängen rechnen, bedingt sind durch die dabei auftretende 
Bei den Durchschneidungs- wie den Reizungsversuchen wurde tibrigens auch auf einen etwaigen Eiweissgehalt des Harns geachtet. Nur in wenigen Proben zeigte sich eine geringe Opalescenz.

Nach Durchschneidung des Halssympathicus scheint kein Autor Diabetes beobachtet zu haben. Pary') gibt sogar ausdrïcklich an, dass sie keine wahrnehmbare Wirkung auf den Harn äussere. Obwohl ich mich bereits bei Thierversuchen gelegentlich von der Richtigkeit der Pavy'schen Angabe iuberzengt hatte, so sollte sie dennoch von Neuem geprüft werden. Zugleich sollte in diesen neuen Versuchen der Durchschneidung des Halssympathicus eine Reizung des Kopfendes nachfolgen. Endlich sollte mit diesen Versuchen eine Prüfung der merkwürdigen Angaben Peyrani's verknipft werden. Peyrani's Versuche scheinen von den Physiologen wenig Berïcksichtigung gefunden zu haben; seine An. gaben sind in keines der physiologischen Handbücher übergegangen. Im Jahresbericht von Henle and Meissner (1870 S. 216) findet sich folgendes kurze Referat: „Nach zahlreichen Versuchen an Hunden, Katzen, Kaninchen findet Peyrani, dass mehrere Stunden fortgesetzte electrische Reizung des Sympathicus am Halse Vermehrung der Harn- und Harnstoffmenge bedinge, Durchschneidung des Sympathicus Verminderung: beider auf ein Minimum bewirke."

Von klinischer Seite haben die Angaben Peyrani's Beachtung gefunden. So sind sie z. B. von Senator ${ }^{2}$ ), dem jenes Referat vorgelegen zu haben scheint, bei Besprechung der Aetiologie und Pathogenese des Diabetes insipidus und vonSeitz ${ }^{3}$ ) bei derselben Gelegenheit erwähnt worden. Wir stützen uns auf die von Peyrani selbst herrührende Mittheilung seiner Untersuchungen in den Comptes rendus ${ }^{4}$ ) und lassen dieselbe absichtlich,

Gefäss-Dilatation und Contraction." Hinsichtlich der weiteren Schlussfolgerungen muss auf das Original verwiesen werden.

1) F. W. Pavy: Untersuchungen über Diabetes mellitus, dessen Wesen und Behandlung. Deutsch von Langenbeck. 1864. S. 81.

2) v. Ziemssen's Handbuch der speciellen Pathologie und Therapie XIII, 2. Hälfte. S. 263.1876.

3) Niemejer-Seitz, Lehrbuch der spec. Path. u, Therap. II. 2. Abth. S. 873 u. 874.1877.

4) Compt. rend. T. 70. p. 1300. 1870. 
um Missverständnissen vorzubeugen, wörtlich folgen, um so mehr, da sie nur kurz ist:

„Dans le but de constater le rôle du grand sympathique dans la sécretion urinaire, j'ai fait une longue série de recherches expérimentales sur vingt-cinq chiens, trois chats et quarante-sept lapins. Dans ces expériences, j’ai évalué la quantité de l'urine et de l'urée, soit dans les six heures qui précédaient toute expérimentation, soit pendant les six heures de l'excitation galvanique (obtenue au moyen d'un courant continu ou d'induction, faible, de force moyenne ou très-fort) du sympathique au cou, mais non coupé, soit pendant les six heures de galvanisation du bout périphérique du sympathique coupé au cou, soit encore pendant les six heures que j'ai observé les animaux après la section au même point du sympathique, sur lequel je n'avais fait arriver aucune excilation.

Voici les conclusions qui en ressortent, dans leur ensemble:

1. Les quantités de l'urine et de l'urée s'élèvent, a u fur et à mesure qu'on augmente la force du courant voltäque.

2. Lorsque l'on emploie des courants galvaniques de la même intensité, le courant d'induction produit une élévation beaucoup plus grande dans la quantité des urines et de l'urée, que le courant constant.

3. Si l'on coupe le sympathique, mais qu'on ne l'excite pas au moyen du galvanisme, la quantité de l'urine et de l'urée atteint un minimum.

4. Lorsque l'on galvanise le bout périphérique du sympathique coupé au cou, la quantité de l'urine et de l'urée descend bien au-dessus du nivean normal, quoique les chiffres soient toujours beancoup au dessous de ceux, qu'on obtient en galvanisant le sympathique qui n'a pas été préalablement coupé."

Ob noch eine weitere ausfiuhhlichere Mittheilung, die entsprechend dem Interesse des Gegenstandes sowie behufs Controlirung der Angaben erwiunseht gewesen wäre, existirt, haben wir trotz ausgiebiger Nachforschnng nicht ermitteln können.

Die verwandten Kaninchen wurden bei ein und demselben Futter in einem Kasten gehalten. Vom Beginn des Versuches an blieben die Thiere ohne Nahrung. Im Uebrigen war die Einrichtung der Versuche folgende: Zunächst wurde der Harn sorgfältig 
ausgedriickt und die Grösse der Harnsecretion bei intactem Sympathicus vier Stunden hindureh bestimmt, jedoch so, dass alle zwei Stunden der Harn ansgedrïckt wurde. Daranf wurden der rechte oder der linke oder beide Halssympathici durehschnitten, die Hautwunde geschlossen and wiederum die Menge des in vier Standen secernirten Harns in derselben Weise bestimmt. Endlich wurde das Kopfende des durchschnittenen Sympathicus (rechts, links oder auf beiden Seiten) eine Stunde lang intermittirend gereizt und in gleicher Weise die Harnmenge vier Stunden hindurch bestimmt. Wir bedienten uns nur des Inductionsstromes (d u Bois'scher Schlittenapparat) und in allen Fällen ein und derselben Stromstärke, wie wir sie beim Vagus und Ischiadicus angewandt hatten. Von den sechs Reizungen, die innerhalb der einen Stunde vorgenommen wurden, dauerte jede einzelne $1 \frac{1}{2}$ Minute.

Um des Effectes vollkommen sicher zu sein, wurde übrigens bei jeder einzelnen Reizung das Verhalten der Pupille beobachtet. Die Thiere wurden nur, so lange es durchaus nöthig war, d. h. behufs der Durchschneidung und der Reizung des Sympathicus gefesselt gehalten. Sämmtliche Harnproben wurden auf Zucker untersucht. Von den zehn angestellten wad glatt abgelanfenen Versuchen haben wir sechs in der Tabelle zusammengestellt. Die übrigen vier liessen sich desshalb nicht wohl der Tabelle einreihen, weil sie in der Versuchsdaner differirten.

Aus der umstebenden Tabelle geht hervor, dass nach der Durchschneidung nur in zwei Fällen eine leichte Reduction im Harn auftrat. In den vier uibrigen, in dieser Tabelle nicht enthaltenen Versuchen gab der Harn in zwei Fällen nach der Durchschneiduug eine deutlichere Zuckerreaction. Nach der Reizung wurde in drei Fällen der Tabelle Zuckerausscheidung eonstatirt, ebenso in drei von den vier oben erwähnten Versuchen. Hervorgehoben sei noch, dass in denjenigen Versuchen der Tabelle, in welchen der Harn nach der Durchschneidung keine Zuckerreaction gab, derselbe auch nach der Reizung mit einer Ausnahme frei davon blieb. 
E. Külz:
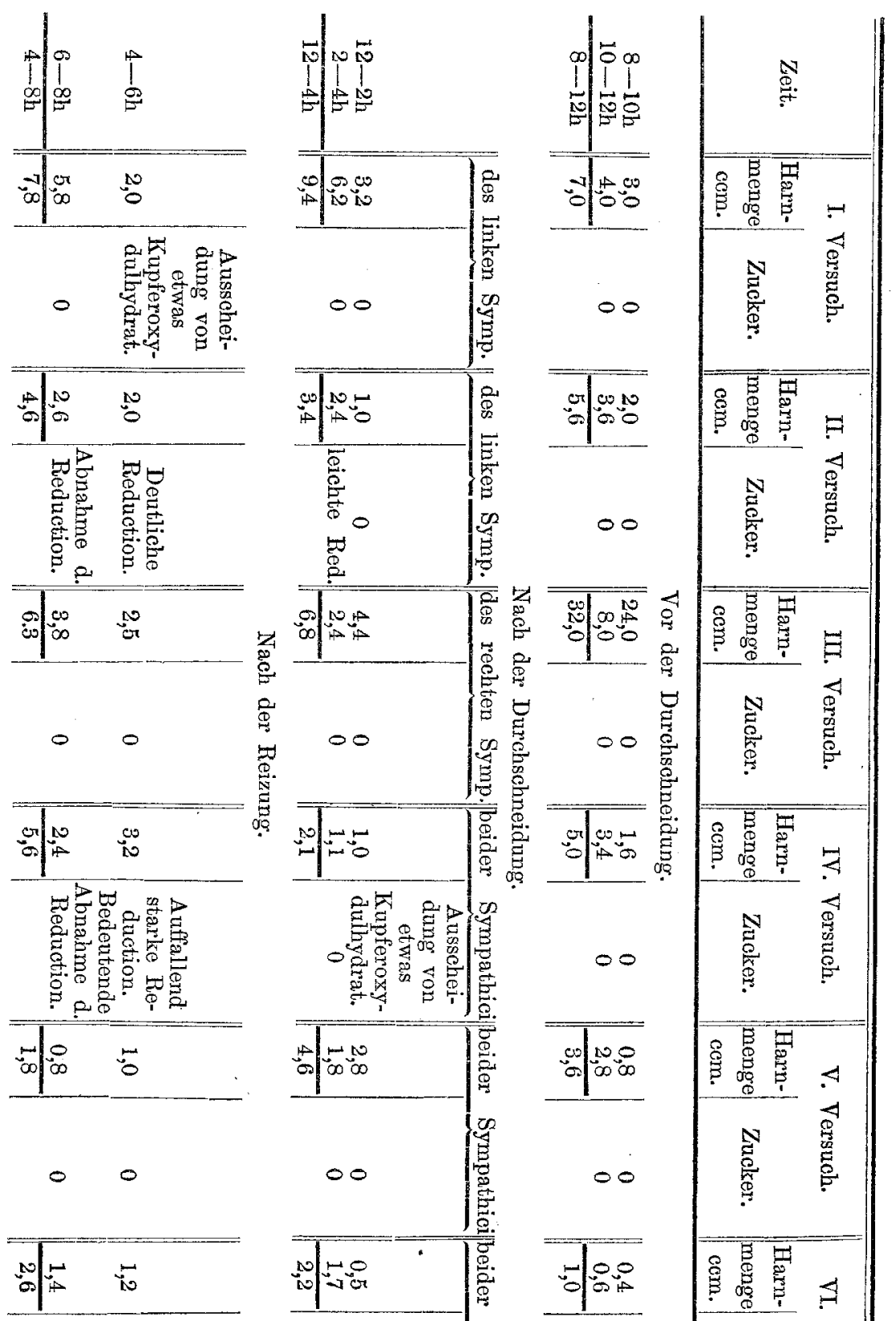

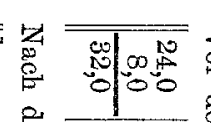

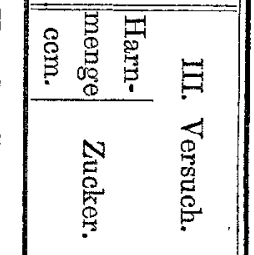

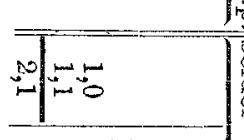

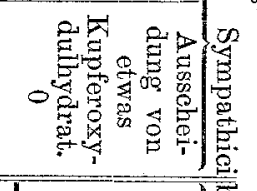
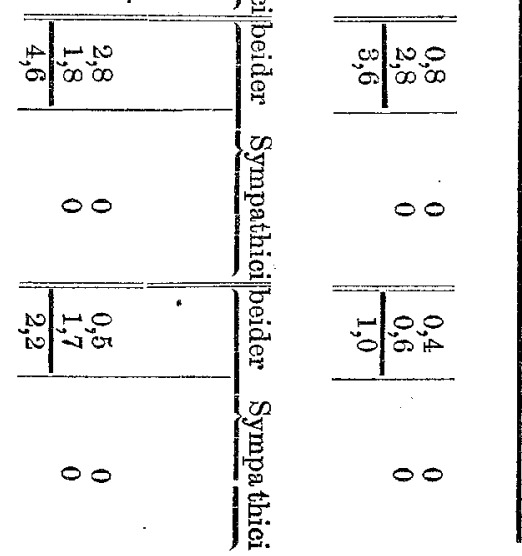

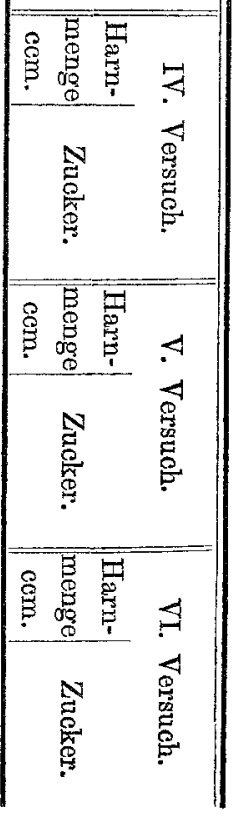


Uebrigens war die Zuckerausscheidung, wo sie überhaupt nach Durchschneidung des Sympathicus auftrat, in keinem Fall so evident, wie nach der Vagusdurehschneidung. Dasselbe gilt auch, wenn anch im geringeren Grade, im Durchschnitt von den Reizungsversuchen.

Was nun die Peyrani'schen Angaben betrifft, so sind wir uns der Verschiedenheit seiner und unserer Versuche wohl bewusst. Während er an Hunden, Katzen und Kaninchen operirte, beschränkten wir uns nur nach Analogie des dieser Arbeit zu Grunde liegenden Untersuchungsplanes auf das Kaninehen. Er verwandte bald den constanten, bald den unterbrochenen Strom mit variabler Stromstärke, wir bedienten uns nur des unterbrochenen Stromes und ein und derselben Stromstärke, wie sie sich uns bei den ibrigen Versuchen hinreichend erwiesen hatte. Er reizte den intacten Sympathicus, während wir das Kopfende des durchschnittenen Sympathicus reizten. Wir liessen analog den Versuchen beim Vagus die Durehschneidung der Reizung vorausgehen, während er umgekehrt die Durchschneidung der Reizung folgen liess. Die gesammte Versuchsdauer erstreckte sich bei uns auf 12 Stunden, bei Peyrani auf 18 Stunden.

Aus diesen Gründen sind selbstverständlich unsere Versuchsresultate mit denen Peyrani's nicht deutlich vergleichbar, um so weniger als Peyrani gar keinen Beleg mittheilt.

Wir sind nicht im Stande, aus dieser Tabelle bei unserer Versuchsform einen bestimmten Einfluss der Durchschneidung des Halssympathicus oder der Reizung seines Kopfendes auf die Harnsecretion herauszulesen, um so weniger, als auch die vier uibrigen Veruche das gleiche Resultat ergaben.

Die in der vorstehenden Arbeit gemachten Angaben beruhen auf 47 Versuchen, über welche die ausführlichen Protokolle vorliegen.

In Wirklichkeit beruhen diese Angaben auf einer weiteren mehr als doppelt so grossen Anzahl von Versuchen, die schon früher gelegentlich, im Ganzen mit denselben Resultaten angestellt wurden. Es kam mir darauf an, dass die einzeinen zu verschiedenen Zeiten gewonnenen Versuchsresultate noch einmal im Zusammenhang methodisch geprift wurden. Die Versuche von Sch iff habe ich 1872 und 1874 wiederholt, ohne jedoch zu ent- 
scheidenden Resultaten zu gelangen. Ich konnte mich desshalb aueh nicht entschliessen, eine bestimmte Ansicht zu äussern. Möglicherweise hat an diesen zweifelhaften Resultaten der Umstand die Schuld getragen, dass ich der Wahl der Versuchtshiere damals nicht diejenige Berücksichtigung geschenkt habe, wie sie mir weitere Beschäftigung mit dem Gegenstand in Uebereinstimmung mit den Angaben Eckhard's für derartige Versuche als wesentlich erscheinen liess ${ }^{1}$ ).

1) I. Ryndsjun (Diabetes mellitus bei Ischias und Ischiadicus-Verletzung. Dissert. Jena 1877) hat Schiff's Angaben einer Prüfung unterzogen. Seine ebenfalls an Kaninchen angesteliten Versuche (Quetschung des Ischiadicus mittelst Ligatur oder Pincette. Durchschneidung eines oder beider Ischiadici, Durchziehung eines in Crotonöl getränkten Fadens, Einspritzung von Sol. Fowleri in die Nervenscheide) führten zu negativen Resultaten. Nur in 2 Versuchen und zwar nach Durchziehung eines mit Crotonöl getränkten Fadens constatirte er vorübergehenden Zuckergehalt des Harns. - In 2 Fällen einer acuten und einer chronischen Ischias fand $R$. den Harn zuckerfrei.

Böhm und Hoffmann (Archiv für experiment. Path. u. Pharmakol. 8, 302) sahen bei Katzen nach rascher Durchschneidung eines oder beider Nerv. ischiadici unzweifelhaft Diabetes auftreten. Allerdings fügen sie hinzu, auch Ischiadionsdurchschneidung ausgefüht zu haben, auf welche kein Diabetes folgte. Der Eingriff führt also nicht unfehlbar zur Glycosurie, deren Ursachen sie in der starken sensiblen Reizung suchen.

F. Froning (Versuche zum Diabetes mellitus bei Ischias. Dissert. Göttingen 1879) erhielt durch Aussehneiden eines Stückes des Nerv. ischiadicus, durch heftige Reizung desselben mittelst dauernder Ligatur, Carbolsäure, Fowler'scher Lösung, Kaliumbichromat bei Kaninchen, Meerschweinchen, einer Katze und einer Hündin zweifellose Meliturie, welche mehrere Tage anhielt. 